\title{
Agent-based Bicycle Traffic Model for Salzburg City
}

\author{
Gudrun Wallentin and Martin Loidl \\ Department of Geoinformatics - Z_GIS, University of Salzburg, Austria · \\ gudrun.wallentin@sbg.ac.at
}

Full paper double blind review

\begin{abstract}
Bicycles play an increasingly important role in sustainable traffic strategies of urban areas. Recent research thus strives to contribute to a better understanding of the spatio-temporal distribution of cycle trips and emerging cycle traffic flow patterns. In this paper we use an agent-based modelling approach to simulate cycle traffic of the city of Salzburg. The presented model specifically aims to test the role of the city's surrounding area for inner-city cycle traffic. Simulation results are validated against data from cycle counting stations. The main results suggest that commuters from outside the city greatly influence the spatial distribution of traffic flows in the city. The agent-based modelling approach thereby proved to be a useful alternative to more conventional transportation modelling methods, not least because its intuitive parameterisation allows for explorative system analysis. Finally, we present a number of open issues to highlight important topics for future research.
\end{abstract}

\section{Introduction}

Knowing when and where, how many and which cyclists are on the road is of great importance for a variety of application contexts: from infrastructure planning to traffic management and accident analysis, just to name three prominent examples. However, as yet only few cities have a system of evenly distributed counting stations across their street network, and are thus able to accurately estimate current bicycle flows. Most cities run only a few stations at known hotspots. The same holds true for Salzburg, which we use as our study site in this work. Due to the small sample size of locations it is hardly possible to estimate city-wide distributions and spatial flows of bicycle traffic with conventional models, such as the traffic demand model (CASCETTA 2001). The lack of data on bicycle trip patterns thus hampers sound analysis, and consequently a better understanding of mechanisms behind bicycle usage (VANDENBULCKE et al. 2011). However, the significant role of cyclists in sustainability strategies for urban transportation (MESCHIK 2012) requires focusing research efforts on the use of bicycles in cities and resulting cycling trip patterns.

In agent based models, the system-level flow patterns emerge from individual human behaviour. Agent-based transportation models are therefore particularly well suited to generate and test hypotheses on individual behaviour. Additionally, simulated individual behaviour can be aggregated to a higher scale level, and thus form the basis for a global estimation of flows (for a general introduction see BAZZAN \& KLÜGL 2014). In the present paper, we apply these concepts to urban cycle traffic. For this we develop a prototype model for cycle traffic in Salzburg, and validate the model with available counting data. 
The aim of this paper is to present the Salzburg Bicycle Model as an agent-based approach, to simulate the spatio-temporal distribution of cyclists across the road network of the city of Salzburg. In particular, we contrast two scenarios of the Salzburg Bicycle Model to test the hypothesis that the spatial distribution of cycle trips in Salzburg can only be understood in relation to its surrounding area. By this example, we aim to evaluate our current level of understanding of the cycle traffic in Salzburg, and to assess the suitability of an agent-based modelling approach to advance this understanding. The proposed Salzburg Bicycle Model is thus intended as a tool to generate new and test existing hypotheses on cyclist behavior, and identify main steps for further research.

\section{Salzburg Bicycle Model}

The Salzburg Bicycle Model reflects our current understanding of cycle traffic in Salzburg. Input parameters were thus exclusively based on available statistical data, findings from literature, and expert inputs; no data-driven calibration was involved. The parameters of interest comprised different, pre-defined types of cycling agents, their relative share of total cyclists, and their respective characteristics. The model is implemented in the agent-based modelling framework NetLogo (WILENSKY 1999).

Two scenarios were designed to test the hypothesis on the importance of Salzburg's surrounding area for the spatial distribution of cyclists: Scenario 1 - "Salzburg City" simulated one day of cycle traffic in the administratively delimitated city of Salzburg $\left(65 \mathrm{~km}^{2}\right)$. Commuters from or to locations beyond the city boundaries were not considered. The city was thus treated as a closed system. The second scenario - "Salzburg City Region" additionally included the greater region of Salzburg with a total area of $330 \mathrm{~km}^{2}$. The conceptual model and all its parameters, including the total amount of simulated cyclists, the characteristics of the street network, and routing preferences were equal in both scenarios.

The simulation results for both scenarios were validated with cyclist counting data from three counting stations. The day that was used for validation was selected randomly, based on four criteria: (1) the validation day represents the warm season of the year, where most cyclists are expected, (2) it further avoids the summer break at universities between 1st July and 30th September to properly account for student cyclists, (3) each of the three counting stations operated correctly, and (4) the weather was suitable for cycling. Finally, Thursday 06 June 2013 was selected. At this date, the maximum temperature was slightly above $22^{\circ} \mathrm{C}$ and no precipitation was recorded, according to the freely available weather archive from www.wetteronline.de ${ }^{1}$.

The following description of the conceptual design of the Salzburg Bicycle Model follows the ODD protocol (GRIMM et al. 2006, GRIMM et al. 2010).

\subsection{Purpose}

The purpose of the model was to simulate the spatio-temporal distribution of cyclists in the

$1 \mathrm{http}: / /$ www.wetteronline.de/wetterdaten/salzburg?period=4\&month=06\&year=2013 [accessed 2014-11-10] 
city of Salzburg during a weekday in late spring. The spatial distribution of the cycle trip density across the city's street network was expected to emerge from the behaviour of individual cyclists. The model was thus intended to be used as a base model to generate and test hypotheses on driving factors of cycle traffic and their implications for traffic management, route preferences, and effects of cycling infrastructure or the identification of accidentprone sites.

\subsection{Agents, State Variables And Scales}

Agents in this model were individual cyclists who travel on the street network of the city of Salzburg. There were three different agent types: 'working cyclists', 'student cyclists', and 'leisure cyclists'. Each agent was assigned two trips: it commutes from home to its respecttive destination and back again. Regardless their type, agents cycled at an average speed of $20 \mathrm{~km} / \mathrm{h}$.

Cyclists preferred the safest route (LOIDL \& ZAGEL 2010) over the shortest route. However, individual preferences and variations in the perception or location awareness with regards to route safety were accounted for by a stochastic variation in routing weights.

The system state of interest was the spatio-temporal distribution of cyclists. The temporal extent of a simulation was one weekday from midnight to midnight the next day, with a resolution of one minute. Cyclist counts at three stations in the city centre served as the state variables to measure and validate the distribution of cyclist over space and time. The counting data had a temporal granularity of 15 minutes. The simulation results were further aggregated over the entire day for each street segment at the end of one simulation run.

\subsection{Design Concepts}

A spatial pattern of cycle-traffic loads across the city of Salzburg emerged from trips of individual cyclists. In its base version, the model's agents did not sense their environment, they do not have a memory of prior experiences, they do not interact with other cyclists and they are not adaptive to their environment. However, all of these behavior types are candidates to be further tested and calibrated in scenarios. Especially interesting results for planning purposes and infrastructure measures were expected to be emergent clusters of frequently traversed street segments, so called hot routes (TOMPSON et al. 2009), and the detection of bottlenecks. Further, the overall result of the simulation was expected to serve as a statistical population for the analysis of discrete, network-bound incidents.

\subsection{Initialisation and Input Data}

Each cyclist generated the starting location by random selection from a resident density grid of $250 \mathrm{~m}$ x $250 \mathrm{~m}$ resolution ${ }^{2}$. Target locations differed between cyclist types: working cyclists have a destination, which is generated randomly from a workplace density grid. Student cyclists drew their targets from a grid of the same resolution that had been computed from university locations and the respective number of students. Leisure cyclists selected destinations preferably within residential areas - which of course, is a vast simplification.

2 If not indicated otherwise, all statistical data were retrieved from Statistics Austriawww.statistik.at [accessed 2014-10-13] 
The parameterisation represented cycle traffic on a day in June, at which a maximum number of cyclists could be expected. The relative share of cyclist types was deduced as follows: In the city of Salzburg there are 64,000 residents who work or study in the city. Of these 64,000 inner-city commuters, the share of students is about 15,000; the remaining 59,000 are thus employees. The city's current modal split of cyclists is $16 \%$ on average (GUMPINGER et al. 2010), where the modal split for students is assumed to be significantly higher (KOK 2013). Consequently, the expected amount of 'working cyclists' was $16 \%$ of 59,000 ( $=9,440$ cyclists), and the amount of 'student cyclists' was $30 \%$ of $15,000(=4,500$ cyclists). The share of work-related traffic was estimated to be $44 \%$ for the motorized private transport in Switzerland (VRTIC et al. 2007); similar figures are reported for Austria by several authors (see ILLEK \& MAYER (2013) for a summary). For a first parameterisation of cycle traffic in Salzburg we assumed $44 \%$ of all trips as being work related. The remaining $56 \%$ thus split between students $(4,500=21 \%)$ and leisure cyclists $(7,510=35 \%)$, who pursued leisure and shopping activities. In total, the amount of simulated agents was 21,450 cyclists per day.

The temporal distribution of trips was modelled stochastically. Starting and returning times were chosen randomly by each individual cyclist, following normal distributions (Fig. 1): working cyclists start in the morning around $7 \mathrm{am}$ (std 60min) and return home after about eight hours of work (std 120min). Students cycled to university around 11:30 am (std 180 $\mathrm{min}$ ) and returned home after about three hours (std 120min). Trips to leisure activities were scheduled to start either at 2pm, with a large standard deviation of four hours, or at $7 \mathrm{pm}$ (std 90min).

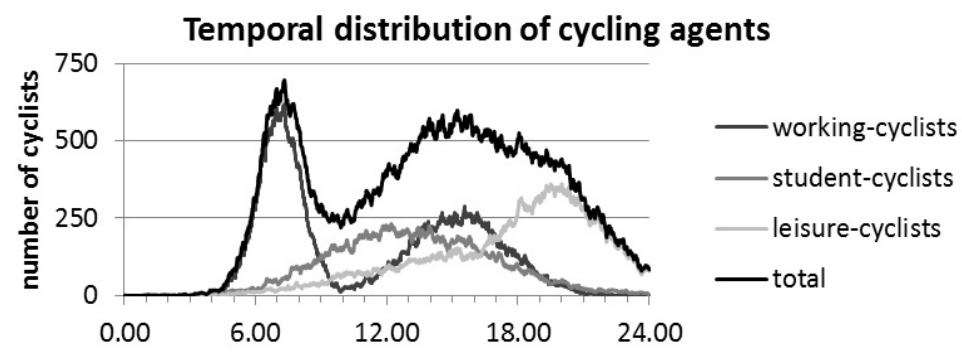

Fig. 1: Temporal distribution of actively cycling agents over one day

The geometry of street segments and their respective attributes was derived from the authoritative road network dataset, the Graph Integration Platform (GIP). Each street segment is associated with information on its length, routing weights, legal restrictions and regulations. In the NetLogo software, these street segments were then rearranged to a routable network dataset. Routes were calculated using the Dijkstra algorithm of NetLogo's network extension, weighted by a street-segment safety index (LOIDL \& ZAGEL 2010). 


\section{Results and Validation}
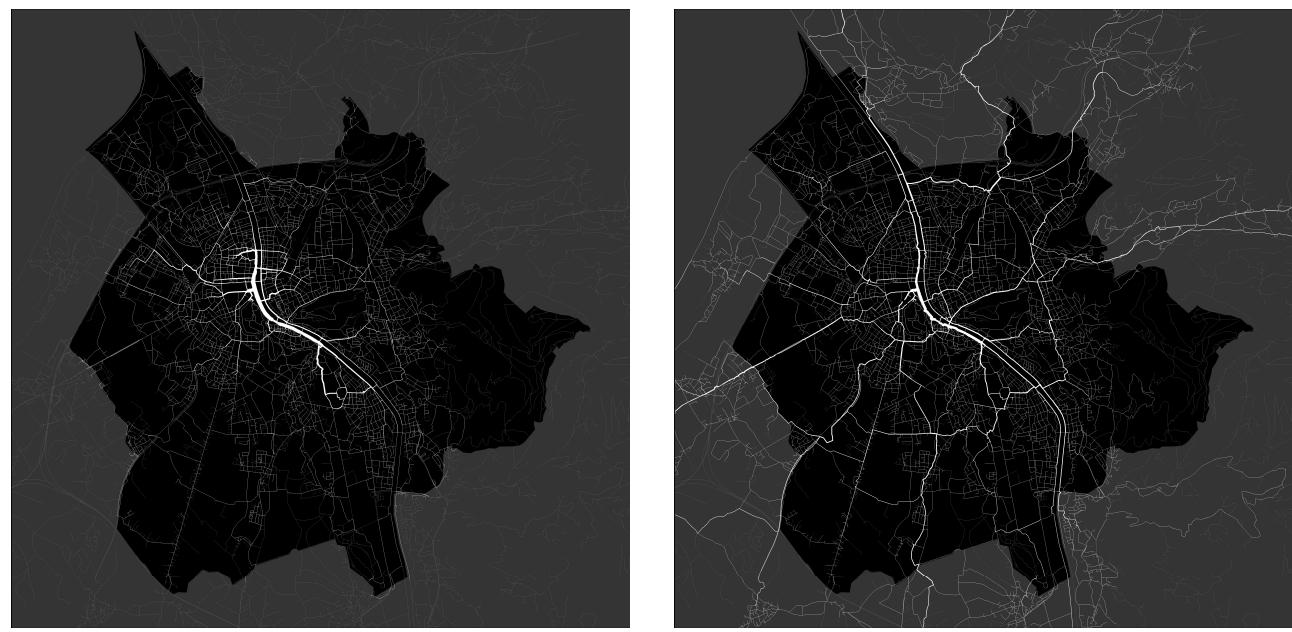

Fig. 2: Heatmaps of simulated bicycle trips: scenario "Salzburg City" (left) and the scenario "Salzburg City Region" (right). The administrative boundaries of Salzburg are highlighted in black.

Fig. 2 visualises emergent cycle traffic flow patterns of the two simulated scenarios as heatmaps. Both scenarios plausibly showed that the cycle ways along the Salzach river are the main corridors for North-South traffic through the city. A number of radial access routes connect this corridor with other parts of the city. Whereas the "Salzburg City" scenario resulted in a strong cluster of cycle trips in the Inner City, the trips in the "Salzburg City Region" scenario were distributed more evenly across the city. Additionally, a cycle ring road emerged that connects city districts around the centre. This 'strong' pattern (GRIMM \& RAILSBACK 2006) of spatial density patterns across the city, which is at the centre of interest, could not directly be validated with the available counting data, because the counting stations were concentrated in the city centre along the Salzach river.

However, a number of 'weak' patterns indicated large differences between the scenarios on the one hand, and a better fit of the "Salzburg City Region" scenario on the other. Weak patterns describe patterns in the simulation results that do not lie in the primary interest of the modeller, but, in combination, can be strong indicators for the structural validity of the model. Such a weak pattern was, for exam-

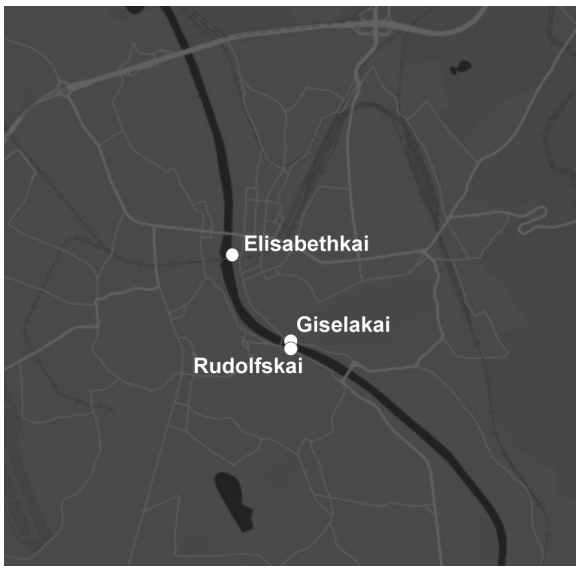

Fig. 3: Location of the counting stations 
stations in the Inner City ("Giselakai" and "Rudolfskai", see Fig. 3) to the slightly more offcentre station "Elisabethkai": whereas the counts of the Inner City stations topped the list with roughly the same order of magnitude, the more remote station comes in third place (Fig. 3). This pattern was reproduced by the "Salzburg City Region" scenario, but not by the "Salzburg City" scenario. Another weak pattern was the ratio of counted cyclists at the opposing counting-stations to the right "Giselakai" and the left "Rudolfskai" of the Salzach river in the Inner City: in the "Salzburg City" scenario this ratio was 1:3.51, whereas the "Salzburg City Region" scenario resulted in a more valid ratio of 1:1.27 with respect to the ratio of 1:0.85 on the validation day, the $6^{\text {th }}$ of June 2013.
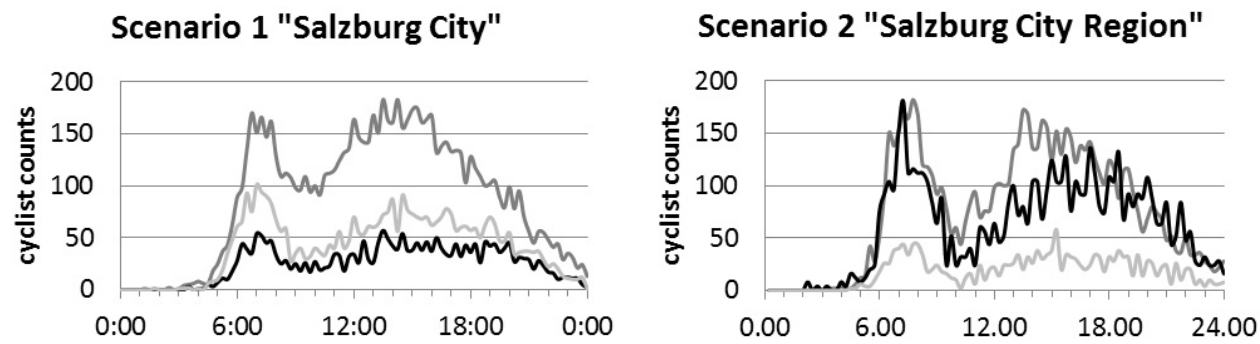

Reference day, Thu 6-jun-2013
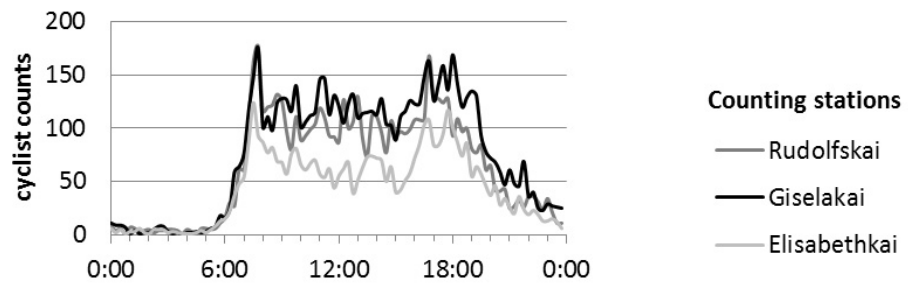

Fig. 4: Cyclist counts for three counting stations for the simulated scenarios (top) and the validation reference day, 6th June 2013 (bottom)

The sum of counted cyclists on both river sides in the inner city amounts to 13,623 trips in the validation data, compared to 10,861 trips ("Salzburg City" scenario), and 6,562 trips ("Salzburg City Region" scenario) in the simulation. The total number of 21,450 modelled cyclists thus indicated that the true number of cyclists within Salzburg on a weekday in early summer amounts to 27,000 (19\% of residents), and to 44,500 cyclists respectively for the entire Salzburg City Region (24\% of residents). At rush hours, the number of cyclists on busy cycle paths reaches about 150 cyclists per 15 minutes (Fig. 4), or about one cyclist every 6 seconds. This pattern corresponded in both scenarios, and also matched well with the validation data.

The temporal distribution of simulated bicycle trips correctly showed two maxima, one in the morning and one in the afternoon. In the morning, cycle traffic starts with an abrupt increase of trips, and gradually decreases in the evening. However, the strong depression before noon doesn't correlate with the validation data. This might point to a further important cyclist type or behaviour that was not accounted for in the present model. 


\section{Discussion and Conclusions}

The results of this research show that bicycle flow patterns on the scale level of the entire city of Salzburg are truly emergent phenomena. As in any complex system, higher level flows ('spatio-temporal patterns') could not be intuitively anticipated from the conceptual model.

Our purpose of modelling cycle traffic in Salzburg by means of agent-based simulation was twofold: First, we aimed to test the hypothesis that a city's surrounding area is an important factor for the spatial distribution of cycle traffic in the city. Towards this end, the results clearly demonstrated that the extent of the study region has a huge impact on the spatial distribution of cycle traffic. In the case of Salzburg, its suburbia is decisive for understanding cycle traffic within the city. Second, we used this example as a suitability test for the agent-based approach to model spatio-temporal flows of cycle traffic on the scale of a city. Recently, the agent approach in which individual agents interact with and adapt to each other has gained momentum in traffic modelling (BAZZAN \& KLÜGL 2014). This development is closely related with 'smart' city initiatives, in which smarter systems emerge from digitally networked and adaptive individuals (BATTY et al. 2012). The agent perspective turns concepts upside down from an abstract view on aggregated populations to a more intuitive level of human beings and their behaviour. MiLLINGTON et al. (2012) therefore refer to agent-based models as "narratives". These narratives are like (complex) hypotheses about what we think to know about the structure of a system and its individual entities, which need to be tested against real-world data. Our narrative on Salzburg's cycle traffic resulted in patterns that matched trip-counting data reasonably well. We therefore conclude that our narrative on Salzburg cyclists tells a realistic story. However, further scenariobased research with the presented Salzburg Bicycle Model is required to develop a sound understanding of the system and further fine-tuning of the model.

Another important aspect in advancing the model relates to empirical validation. For obvious reasons, no simulation model can be more accurate than the data that were used to validate it (WALLENTIN \& CAR 2012). Currently, the spatial distribution of cycle counting stations strongly focuses on the central parts of the city. Therefore the validation data do not provide a representative picture of how the city's network of bicycle infrastructure is used. However, the model can help identify relevant locations for counting stations to sample useful validation data.

Further research effort is required for the following aspects:

- The spatial delimitation of the model system turned out to be crucial. Thus it needs to be further investigated how to define thresholds for the definition of system boarders.

- The three pre-defined cyclist types do not imperatively represent the factual composition of user groups. This composition, together with information concerning the respective trip purpose, average trip length etc., needs to be defined more precisely.

- Until now, the agents are "blind" to their environment and other agents. The implementation of responsive and adaptive behaviour might contribute to a more accurate model.

- Additional counting stations, which are distributed more evenly in the network, would enhance the validation data and thus the model quality. 
As was demonstrated, the agent-based approach generates plausible results, even under very simplified conditions and with reasonable parameterisation effort. Thus it is a promising alternative to existing traffic model approaches and could contribute to a better understanding of urban cycle traffic, based on individual behaviour. After further refinements and calibration the presented model should be suitable to form the basis for applications that build on the estimated cycle flows.

\section{References}

Batty, M., Axhausen, K. W., Giannotti, F., Pozdnoukhov, A., Bazzani, A., Wachowicz, M., Ouzounis, G. \& Portugali, Y. (2012), Smart cities of the future. The European Physical Journal, Special Topics 214 (1), 481-518, doi:10.1140/epjst/e201201703-3.

BAZZAN, A. L. C. \& KLÜGL, F. (2014), A review on agent-based technology for traffic and transportation. The Knowledge Engineering Review 29 (03), 375-403. doi:10.1017/S0269888913000118.

CASCETTA, E. (2001), Transportation systems engineering: theory and methods. Springer.

Grimm, V., Berger, U., Bastiansen, F., Eliassen, S., Ginot, V., Giske, J., GossCustard, J., Grand, T., Heinz, S. K., Huse, G., Huth, A., Jepsen, J. U., Jørgensen, C., Mooij, W. M., Müller, B., Pe’er, G., Piou, C., Railsback, S. F., Robbins, A. M., Robbins, M. M., Rossmanith, E., RÜger, N., Strand, E., Souissi, S., Stillman, R. A., Vabø, R., Visser, U. \& DeAngelis, D. L. (2006), A standard protocol for describing individual-based and agent-based models. Ecological Modelling, 198 (1-2), 115-126. doi:10.1016/j.ecolmodel.2006.04.023.

GrimM, V. \& RAILSBACK, S. (2006), Agent-Based Models in Ecology: Patterns and Alternative Theories of Adaptive Behaviour. In: Billari, F., Fent, T., Prskawetz, A. \& Scheffran, J. (Eds.), Agent-Based Computational Modelling. Physica-Verlag, Heidelberg, 139-152.

Grimm, V., Berger, U., DeAngelis, D. L., Polhill, J. G., Giske, J. \& Railsback, S. F. (2010), The ODD protocol: A review and first update. Ecological Modelling, 221 (23), 2760-2768, http://dx.doi.org/10.1016/j.ecolmodel.2010.08.019.

Gumpinger, G., Thaler, R. \& Eder, M. (2010), Studie - Radfahren und Einkaufen. BUNDESMINISTERIUM FÜR LAND- UND FORSTWIRTSCHAFT, U. U. W. Wien, CIMA Beratung + Management GmbH.

ILleK, G. \& MAYER, I. (2013), Radverkehr in Zahlen - Daten, Fakten und Stimmungen. BMVIT, Wien,

KOK, F. (2013), PLUS-Mobilitätsanalyse 2013. Salzburg: Mobilitätsmanagement der Universität Salzburg.

LOIDL, M. \& ZAGEL, B. (2010), Wie sicher ist sicher? Innovatives Kostenmodell zur Ermittlung des Gefährdungspotenzials auf Radwegen. In: STROBL, J., BlaschKE, T. \& GrIESEBNER, G. (Hrsg.), Angewandte Geoinformatik 2010. Wichmann, Berlin/Offenbach, 394-403.

Meschik, M. (2012), Reshaping City Traffic Towards Sustainability Why Transport Policy should Favor the Bicycle Instead of Car Traffic. Procedia - Social and Behavioral Sciences, 48 (0), 495-504. http://dx.doi.org/10.1016/j.sbspro.2012.06.1028. 
Millington, J. D. A., O’Sullivan, D. \& Perry, G. L. W. (2012), Model histories: Narrative explanation in generative simulation modelling. Geoforum, 43 (6), $1025-$ 1034. http://dx.doi.org/10.1016/j.geoforum.2012.06.017.

Tompson, L., Partridge, H. \& ShePherd, N. (2009), Hot Routes: Developing a New Technique for the Spatial Analysis of Crime. Crime Mapping: A Journal of Research and Practice, 1 (1), 77-96.

Vandenbulcke, G., Dujardin, C., Thomas, I., Geus, B. D., Degraeuwe, B., Meeusen, R. \& PANIS, L. I. (2011), Cycle commuting in Belgium: Spatial determinants and 'recycling' strategies. Transportation Research Part A: Policy and Practice, 45(2), 118-137. http://dx.doi.org/10.1016/j.tra.2010.11.004.

Vrtic, M., Schüssler, N., Erath, A., Meister, K. \& Axhausen, K. (2007), Tageszeitliche Fahrtenmatrizen im Personenverkehr an Werktagen im Jahr 2000. Zürich, Institut für Verkehrsplanung und Transportsysteme (IVT), ETH Zürich, 90 p.

WALlENTIN, G. \& CAR, A. (2012), A framework for uncertainty assessment in simulation models. International Journal of Geographical Information Science, 27 (2), 408-422. http://dx.doi.org/10.1080/13658816.2012.715163.

WiLEnSKY, U. (1999), NetLogo. Evanston, IL., Center for Connected Learning and Computer-Based Modeling, Northwestern University. 